\title{
Electrodynamics study of plasmonic bonding and antibonding forces in a bisphere
}

\author{
Jack Ng, Ross Tang, and C. T. Chan* \\ Department of Physics, The Hong Kong University of Science and Technology, Kowloon, Hong Kong \\ (Received 12 February 2008; revised manuscript received 8 April 2008; published 6 May 2008)
}

\begin{abstract}
Light excitation of surface plasmons in metallic nanoparticle clusters can induce huge forces. We study such forces by using an electrodynamics approach, which fully incorporates the retardation effect. As two particles approach each other, the single-particle plasmons hybridize and split into attractive bonding modes and repulsive antibonding modes, which eventually evolve into an attractive band and a repulsive band. At an intensity of about $0.05 \mathrm{~W} / \mu \mathrm{m}^{2}$, the force can reach nano-Newtons and, hence, can dominate over other relevant interactions.
\end{abstract}

DOI: 10.1103/PhysRevB.77.195407

PACS number(s): 78.67.-n, 42.50.Wk, 73.22.Lp, 78.20.Bh

\section{INTRODUCTION}

Light can exert significant optical forces on small particles, in particular, metallic nanoparticles. ${ }^{1-9}$ This paper investigates the laser induced optical forces of metallic nanoparticle clusters when their localized surface plasmons (SPs) are excited. Such forces are important because they may affect the signal of surface enhanced Raman spectroscopy ${ }^{10,11}$ or promote controllable aggregation of nanoparticles. ${ }^{12,13}$ Two recent papers also studied such forces by using the quasistatic approximation (QSA), ${ }^{12,14}$ but in this paper, we go beyond QSA and employ electrodynamics. The electrodynamics calculation incorporates the retardation effect and is thus more accurate. ${ }^{15}$ We found that the light-induced force calculated by electrodynamics can be interpreted by using QSA, but that the magnitude is smaller than those predicted by QSA, and electrodynamics are also able to "see" the forces induced by the "dark modes" that are also very strong. For a pair of $30 \mathrm{~nm}$ radius nanospheres at a high light intensity and at a particle separation of less than several nanometers, these resonant forces are quite large. They completely dominate over other relevant forces, including the van der Waals attraction.

We will see that the graph of surface plasmon frequency versus particle separation can serve as a road map that helps to explore the properties of the SP induced optical forces. The decrease in particle separation induces hybridization, in which the single-sphere SPs are split into attractive bonding modes and repulsive antibonding modes. Moreover, as a consequence of the strong interaction between the SPs at small particle separations, the attractive (repulsive) modes are significantly forced to shift downward (upward) in frequency, resulting in the formation of a low frequency attractive (high frequency repulsive) band. This is true irrespective of the polarizations, the number of particles, and the particle shape. As a result, low frequency laser illumination will induce strong attraction, which promotes particle clustering, and high frequency illumination will induce strong repulsion, which prevents particle aggregation.

\section{METHODOLOGY}

In our theoretical treatment, we employed the previously developed multiple scattering and Maxwell stress tensor
(MS-MST) formalism (also known as the generalized Lorenz-Mie theory for multi-sphere). ${ }^{16,17}$ For nontouching spheres, the MS-MST formalism is highly accurate and rigorous. It takes the full electrodynamics effect into account without making approximations. ${ }^{18}$ Unless otherwise stated, we use a Drude model for the dielectric function, which is a fitting of the experimental data of bulk silver, ${ }^{19}$

$$
\varepsilon_{r}(\omega)=\varepsilon_{a}-\left(\varepsilon_{b}-\varepsilon_{a}\right) \omega_{p}^{2} /[\omega(\omega+i \gamma)],
$$

where $\varepsilon_{a}=5.45, \varepsilon_{b}=6.18, \omega_{p}=1.72 \times 10^{16} \mathrm{rad} / \mathrm{s}, \quad \gamma=\nu_{F} / l$, $\nu_{F}=1.38 \times 10^{6} \mathrm{~m} / \mathrm{s}$, and $l=52 \mathrm{~nm}$. We shall consider a pair of nanospheres depicted in Fig. 1(c), and we focus on the optical binding force ${ }^{20}\left(\vec{F}_{B}=\vec{F}_{2}-\vec{F}_{1}\right.$, where $\vec{F}_{i}$ is the force on the $i$ th sphere) that acts to attract or repel the particles from each other, ${ }^{16,21}$ with the convention that a positive value of the force indicates repulsion. Following Ref. 12, we assume an incident intensity of $I_{0}=0.05 \mathrm{~W} / \mu \mathrm{m}^{2}$, which can be achieved, for instance, with a focused beam. We note that the optical forces linearly scale with the incident intensity.

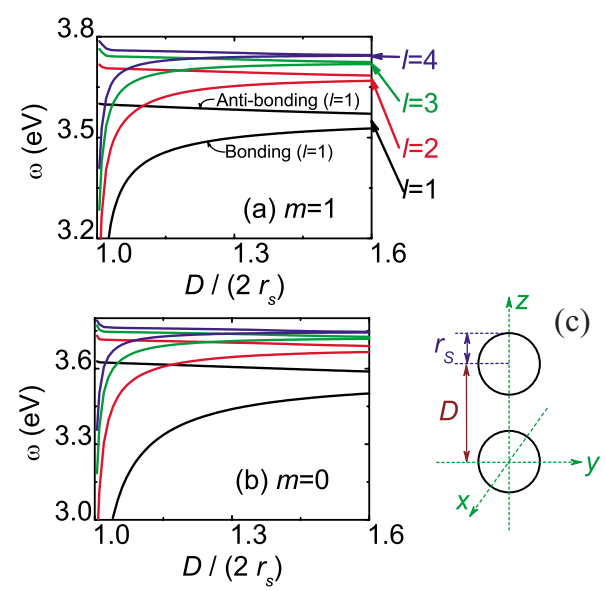

FIG. 1. (Color online) The dimer plasmon frequency (for $l$ $=1-4$ ) versus interparticle separation for a pair of nanospheres as depicted in (c). (a) Electric field polarization perpendicular to the bisphere axis, i.e., $m= \pm 1(m=1$ and $m=-1$ are degenerate). The bonding branch and the antibonding branch for $l=1$ are marked in the figure. The single-sphere plasmon frequency is marked on the right axis. (b) Polarization parallel to the bisphere axis, i.e., $m=0$. (c) An illustration of the geometry under consideration. 


\section{RESULT}

\section{A. Physical picture based on quasistatic approximation}

The natural electromagnetic modes of a metallic nanosphere are its SP modes. When a pair of such nanospheres is within the proximity of each other, owing to the strong interaction, their SP modes will hybridize in a process analogous to the hybridization of atomic orbitals in real molecules. ${ }^{22,23}$ The hybridization and splitting of plasmonic modes are illustrated in Figs. 1(a) and 1(b) for perpendicular $(m= \pm 1)$ and parallel $(m=0)$ polarizations, respectively. Here, perpendicular and parallel are relative to the bisphere axis. For a qualitative understanding of the problem, we used QSA with $\gamma=0$ in Eq. (1) to calculate the SP frequencies in Fig. 1. The details of the method can be found, for example, in Ref. 24. If we use electrodynamics, the SP frequencies are complex numbers that are difficult to display and interpret. We have included modes of order up to $l_{\max }=50$ to ensure convergence for the modes plotted in Fig. 1. Such QSA calculation is not quantitatively correct, but it could provide useful insights. The SP frequencies of a single isolated nanosphere are marked on the right axis of Fig. 1(a). As the nanospheres approach each other, their modes start to weakly interact, and the single-sphere SP modes hybridize and split into a bonding mode and an antibonding mode, with the bonding mode at a lower frequency and the antibonding mode at a higher frequency. The bonding mode and the antibonding mode associated with $l=1$ are labeled in Fig. 1(a). A bonding mode is characterized by having an antisymmetrical field pattern upon reflection from a plane perpendicular to the bisphere axis, while an antibonding mode has a symmetrical field pattern.

Figure 1 can help us qualitatively understand the SP induced optical forces. If we assume that the total electromagnetic energy is dominated by one resonance mode, it can be shown that the resonant optical force due to that mode may be expressed as

$$
F_{B} \approx-\frac{1}{\omega} \frac{d \omega}{d D} U \propto-\frac{d \omega}{d D},
$$

where $U$ is the total electromagnetic field energy and $\omega$ is the frequency of the mode. ${ }^{25}$ We see from Fig. 1 that the bonding modes and antibonding modes have positive $(d \omega / d D>0)$ and negative $(d \omega / d D<0)$ definite slopes, respectively; consequently, they must, respectively, induce attraction and repulsion according to Eq. (2). Moreover, since the force is proportional to the negative of the slope, the bonding mode induced attraction is greater than the antibonding mode induced repulsion in general, owing to the steeper slopes of the bonding modes.

There are three physically distinct frequency regimes: the interval $\Lambda=\left[\omega_{l=1}, \omega_{l \rightarrow \infty}\right]$, the frequency regime below $\Lambda$, and the frequency regime above $\Lambda$. Here, $\omega_{l=1}=3.55 \mathrm{eV}$ and $\omega_{l \rightarrow \infty}=3.81 \mathrm{eV}$ are, respectively, the lowest and highest resonance frequencies for a single sphere. The frequency interval below $\Lambda$ forms an attractive band and the frequency interval above $\Lambda$ forms a repulsive band, whereas both lightinduced attraction and repulsion are possible for the interval $\Lambda$.
In the limit $D \rightarrow \infty$, the bisphere modes are just the singlesphere modes, and hence their resonance frequencies fall inside $\Lambda$. As $D$ decreases, the bisphere resonance frequencies no longer fall inside $\Lambda$. We see from Fig. 1 that the coupling of the modes in the spheres will split each mode into a bonding and antibonding mode pair. The bonding mode has a lower frequency and the antibonding mode has a higher frequency. When the spheres are close together, the bonding modes will have a lower frequency than the minimum frequency of the interval $\Lambda$, and when these modes are excited, they can only give rise to attractive forces. If we apply external light with frequencies below the minimum frequency of the interval $\Lambda$, we can only excite the bonding modes, and thus the light-induced force must be attractive within a QSA picture. The converse is true for the antibonding modes. When the spheres are close apart, they are squeezed to higher frequencies, which can be higher than the maximum of the interval $\Lambda$. External light with frequencies above the maximum of the interval $\Lambda$ will only excite these antibonding modes, and the induce forces are definitely repulsive within a QSA picture.

We see from Fig. 1 that inside the frequency interval $\Lambda$, we can find both bonding modes and antibonding modes. For an incident laser with frequency inside $\Lambda$, these modes can be excited and thus both attractive and repulsive lightinduced forces can be observed in that frequency interval. In this domain, it is possible that a pair of particles can be trapped by a bonding mode and an antibonding mode if the resonant separation of the bonding mode is slightly longer than that of the antibonding mode. In this case, if the distance between the particles is increased, the bonding mode is triggered which pulls the particles back, and if the interparticle distance become smaller, the antibonding mode is triggered which pushes the particles away, thereby trapping the pair of particles to a fixed separation range. We remark that the numerical value of $\omega_{l=1}$ and $\omega_{l \rightarrow \infty}$ presented here are calculated by using QSA; consequently, they do not mean to be quantitatively correct, but the conceptual picture is useful for interpreting some results in the electrodynamics calculations.

\section{B. Electrodynamics calculations}

Our full classical electrodynamics calculation for a pair of $30 \mathrm{~nm}$ radius nanospheres is presented in Fig. 2. Figure 2(a) shows the laser-induced resonant attraction when the attractive band is excited by an incident light with $\omega=3.25 \mathrm{eV}$ $\left(\omega<\omega_{l=1}\right.$ in QSA). The incident light of both polarizations are plotted, with the solid line for parallel polarization and the dashed line for perpendicular polarization. For both polarizations, we observe several attractive resonances as the separation $D$ is varied. These resonant peaks are marked by arrows in Fig. 2(a), and each peak is due to an individual resonance of the bisphere. We note that the peak for $m=0$ at $D /\left(2 r_{s}\right) \simeq 1.06$ is weak and rather difficult to see. In QSA, these attractive resonances correspond to the intersections of the horizontal $\omega=3.25 \mathrm{eV}$ line with the attractive bands in Figs. 1(a) and 1(b). However, for the particle size and separation considered here, the resonant peaks predicted by QSA 

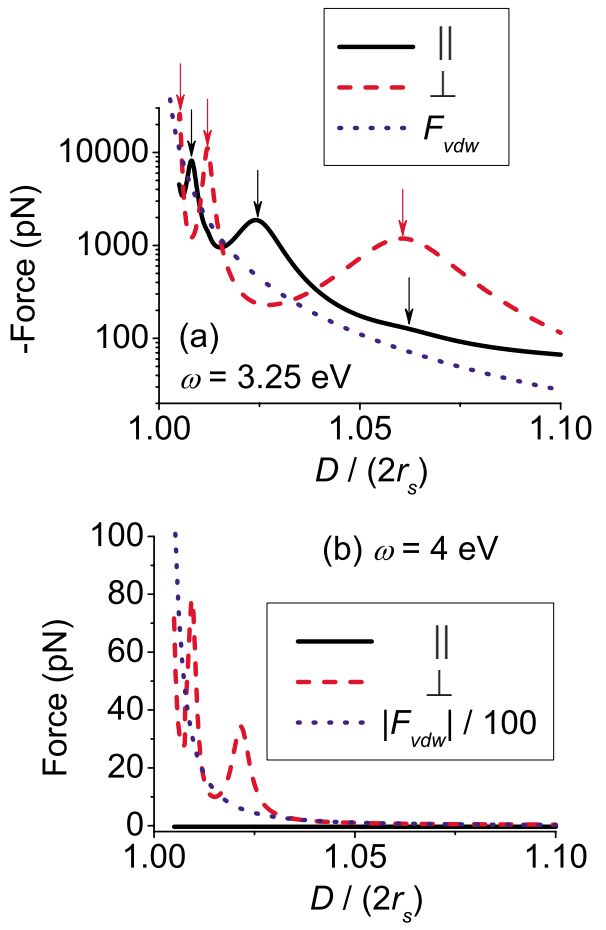

FIG. 2. (Color online) Optical forces as a function of the separation $D . r_{s}=30 \mathrm{~nm}$. Solid line (black): polarization parallel to the bisphere axis (a $z$-polarized and $x$-propagating plane wave). Dashed line (red): polarization perpendicular to the bisphere axis (an $x$-polarized and $z$-propagating plane wave). Dotted line (blue): the nonretarded van der Waals force $F_{\mathrm{vdw}}$. (a) Negative of optical force when the attractive band is excited with $\omega=3.25 \mathrm{eV}$. (b) Optical force when the repulsive band is excited with $\omega=4 \mathrm{eV}$.

deviate from those of the electrodynamics. We note that the forces at the attractive resonances get weaker as $D$ increases. This is qualitatively consistent with the QSA results in Fig. 1 as the slope of the resonance bands generally become smaller at larger distances. The lowest order resonance for $m=0$ should appear at a larger separation than that of $m=1$. However, such mode is not observed even if we extend the separation in Fig. 2(a). One possible reason for this is that the lowest order resonance for $m=0$ is too weak to be distinguishable from the background. The resonant forces are rather strong, on the order of several nano-Newtons at the assumed intensity. However, the range of the resonant force is short, as one can see from Fig. 1. For comparison, the nonretarded van der Waals force $F_{\mathrm{vdw}}=-A r_{s} /\left[12\left(D-2 r_{S}\right)^{2}\right]$, where the Hamaker constant $A=40 \times 10^{-20} \mathrm{~J}$, is also plotted. ${ }^{26}$ It can be seen that for the separation shown (up to $6 \mathrm{~nm}$ ) that the optical force is stronger than the van der Waals force whenever the resonances are excited and hence can dominate the interparticle interaction.

Interestingly, the force induced by the perpendicular polarization is slightly stronger than that of the parallel polarization. It is commonly believed that in order to have a strong optical force, it is necessary to have the "hot spot" (a small region of enormous field enhancement) created by an incident light with parallel polarization. We do observe the hot spot in the parallel polarization. However, our results showed that such hot spot is, in fact, unnecessary, since per-

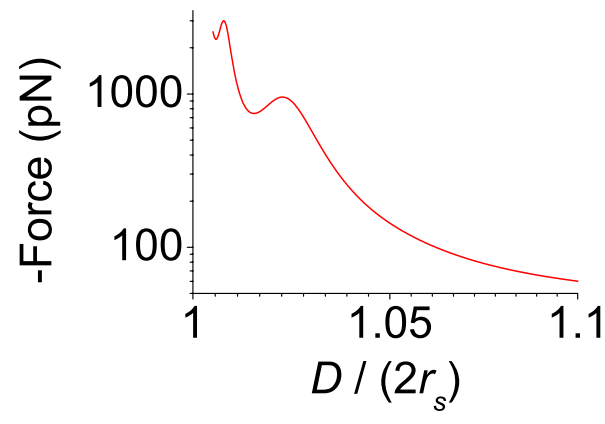

FIG. 3. (Color online) Negative of optical force acting on a pair of $30 \mathrm{~nm}$ radius $\mathrm{Ag}$ nanospheres versus interparticle separation $D$. We used dielectric function of silver measured by Johnson and Christy (Ref. 27). The incident wave is polarized along the bisphere axis (i.e., a $z$-polarized and $x$-propagating plane wave), with $\omega$ $=3.25 \mathrm{eV}$.

pendicular polarization creates no such hot spot, but the induced optical force remains large.

The resonant repulsion associated with the repulsive bands is shown in Fig. 2(b), wherein the incident light frequency is $\omega=4 \mathrm{eV}\left(\omega>\omega_{l \rightarrow \infty}\right.$ in QSA). We shall first consider the perpendicular polarization (dotted line). Only the antibonding modes are excited at this frequency; therefore, several repulsive resonances are observed. The antibonding mode induced repulsions are 2 orders of magnitude weaker than the bonding mode induced attraction shown in Fig. 2(a). This could be understood by noting in Fig. 1 that the antibonding modes have much smaller slopes and therefore a much smaller force according to Eq. (2). The optical force associated with the parallel polarization (solid line) is negligibly small compare to that of the perpendicular polarization. This is because for the geometry we considered, the excitation of the antibonding modes by parallel polarization is forbidden by symmetry. We remark that the $m=0$ polarization also has antibonding modes, but they can only be excited by using an oblique incident wave. We note that the van der Waals force is significantly stronger than the optical force at the assumed intensity. In other words, the repulsive force can play a role only at a very high intensity $\left(\sim 1 \mathrm{~W} / \mu \mathrm{m}^{2}\right)$.

Our electrodynamics calculation can be directly compared to that of the QSA (the most comprehensive results are reported in Ref. 14). At first glance, one might expect the QSA to agree with electrodynamics at least qualitatively, since the length of the entire bisphere system is only $\sim \lambda / 3$. However, it turns out that they significantly differ from each other. Figure 3 shows the resonant optical force acting on a pair of $30 \mathrm{~nm}$ radius $\mathrm{Ag}$ nanospheres illuminated by an incident plane wave with polarization parallel to the bisphere axis (i.e., a $z$-polarized and $x$-propagating plane waves). In order to compare with the QSA of Ref. 14, we used dielectric function of silver measured by Johnson and Christy. ${ }^{27} \mathrm{Com}-$ pare Fig. 3 to Fig. 2(a) of the second reference in Ref. 14, while both results indicate multiple attractive peaks, there are many noticeable differences. First, taking into account the different intensity used in both articles, the QSA calculation gave an optical force higher than the electrodynamics results (by roughly 1 order of magnitude). Second, QSA results indicate that parallel (perpendicular) polarization induced only 


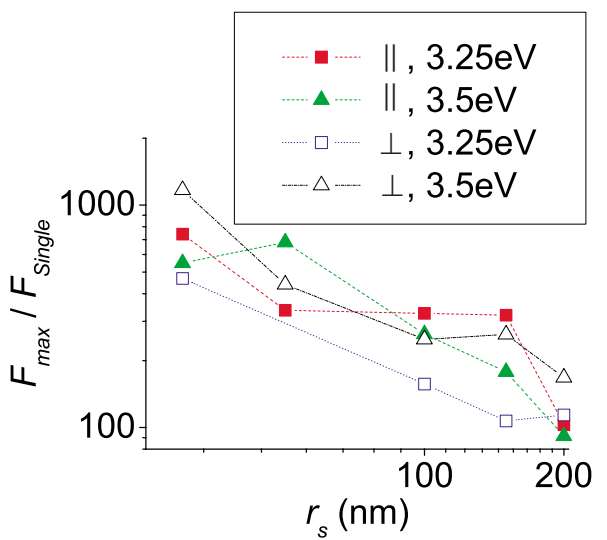

FIG. 4. (Color online) $\left|F_{\text {max }}\right| / F_{\text {single }}$ versus particle size $\left(r_{s}\right)$, where $F_{\max }$ indicates the strength of resonant optical force (see text) and $F_{\text {single }}$ is the single-particle radiation pressure.

resonant attraction (repulsion); however, the electrodynamics result (Fig. 2) shows that depending on the incident frequency, both resonant attraction and repulsion are possible for any polarization. The reason that the resonant attractions (repulsions) for the perpendicular (parallel) polarization are missing in the QSA is because these resonances are the dark modes in electrostatics, which cannot be excited by a uniform electrostatic field owing to symmetry. However, these dark modes can be excited in electrodynamics since the wavelength is finite. Moreover, for the $30 \mathrm{~nm}$ radius nanospheres discussed here, the forces induced by the dark modes are equally strong compared to that of the bright modes (see Fig. 2).

We also recalculated part of the calculation in Ref. 12 on Ag nanospheres. It was pointed out in Ref. 12 that there are three distinct regions in the optical force versus separation curve: they are interparticle separations being much smaller, comparable to, and much larger than the particle size. In the near field region, Ref. 12 reported a rapidly and monotonically increasing attraction as the particle separation decreases. Our electrodynamics result confirmed that the enhanced attraction is of the same type of resonance discussed in this paper. In other words, for separations smaller than those considered in Ref. 12, the force will not monotonically increase as the separation decreases. Instead, the force will reach a maximum and then decline again, just like the resonant peaks in Figs. 2 and 3. Moreover, as $D$ decreases further, other higher order resonances will also be encountered. In the far field region, Ref. 12 reported a monotonically decreasing optical force that scales as $\sim D^{-4}$. Our electrodynamics calculation shows that owing to the retardation, the optical force oscillates between attraction and repulsion, with a magnitude that scales as $\sim D^{-2}$ in the far field, just as for two dielectric spheres. ${ }^{16,21}$

We next explore the size dependency of the resonant optical force. Figure 4 plots $\left|F_{\text {max }}\right| / F_{\text {single }}$ versus $r_{s}$ (radius of the particle) for two incident frequencies $(3.25$ and $3.5 \mathrm{eV})$ and two polarizations. Here, $F_{\text {single }}$ is the radiation pressure exerted on a single nanosphere by an incident plane wave, and $F_{\max }$ is the maximum optical force, at a given frequency and $r_{s}$, which act on a pair of nanoparticles that are separated

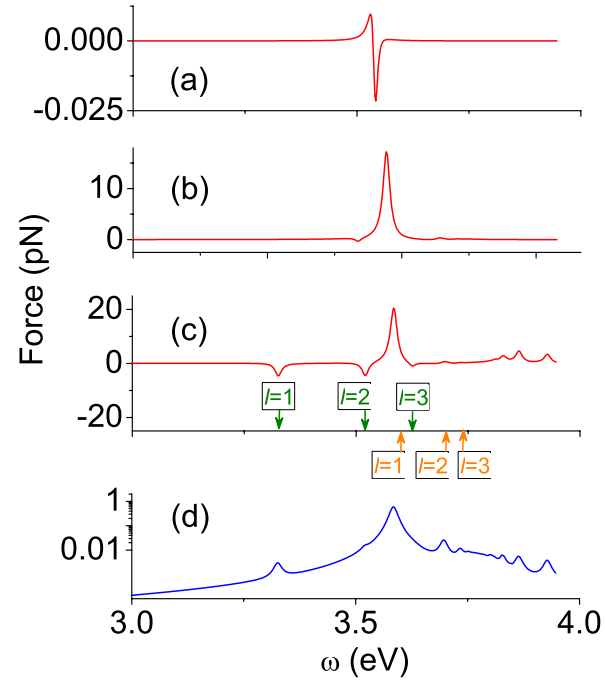

FIG. 5. (Color online) Resonant optical force as a function of frequency for various particle separations. A pair of $5 \mathrm{~nm}$ radius nanospheres is illuminated by an incident wave that propagates along the bisphere axis. (a) $D /\left(2 r_{s}\right)=8$, (b) $D /\left(2 r_{s}\right)=1.4$, and (c) $D /\left(2 r_{s}\right)=1.05$. (d) The total optical force for the configuration in (c).

by more than $1 \mathrm{~nm}$. It is obtained in the following manner. At a fixed $r_{s}$ and frequency, we calculate the optical force for $D \geq 1 \mathrm{~nm}$ to search for the strongest force, which we denote by $F_{\text {max }}$. The ratio $\left|F_{\text {max }}\right| / F_{\text {single }}$ compares the strength of the resonant interparticle force with the single-particle force. It is evident from the curves in Fig. 4 that the resonant interparticle optical force $F_{\max }$ is orders of magnitude stronger than single-particle force $F_{\text {single }}$; nevertheless, their ratio $\left|F_{\text {max }}\right| / F_{\text {single }}$ has a trend to decrease as radius increases.

We now turn our attention to smaller particles. In Fig. 5, the optical forces are plotted against the frequency for a pair of $5 \mathrm{~nm}$ radius nanospheres at several particle separations. The incident light is perpendicularly polarized to the bisphere axis (i.e., an $x$-polarized and $z$-propagating wave). There are three distinct regions in separation that correspond to different mechanisms: they are the surface-to-surface separation being much larger [Fig. 5(a)], comparable to [Fig. 5(b)], and much smaller than [Fig. 5(c)] the particle size. For separations that are large compared to the particle size, as shown in Fig. 5(a) [with $D /\left(2 r_{s}\right)=8$ ], we observe a pair of $(l=1)$ attractive and repulsive peaks, lying adjacent to each other. The force is weak at that regime. The emergence of the pair of peaks can be understood in the following manner. While in the near field regime, the resonant force is dominated by the dipole-dipole interaction, in the far field regime discussed here, the resonant force will be dominated by the interaction of the dipole and the incident field. ${ }^{28}$ The dipole moment is proportional to the polarizability of the particle. The optical force changes sign as the polarizability function changes sign near a resonance. We remark that the type of attractive and/or repulsive pair of force shown in Fig. 5(a) is not limited to the $l=1$ mode; they can also be seen in higher order modes if we use larger spheres. Furthermore, this type of resonant force in the far field is completely different from the bonding and antibonding pair of forces we have previ- 
ously discussed for particles close to each other (for example, those shown in Fig. 2). When the particles are far away, the force is dominated by the product of the incident field and the resonant scattered field, whereas when the particles are close together, the resonant scattered field is actually much larger than the incident field and so that the force is dominated by the quadratic term in the resonant scattered field. For separation comparable to the particle size $\left[D /\left(2 r_{s}\right)=1.4\right]$ shown in Fig. 5(b), the modes start to more strongly interact. To a first approximation, the modes of the individual nanospheres with the same $l$ and $m$ hybridize, which form dimer plasmons of bonding (even) and antibonding (odd) combinations. ${ }^{29}$ This is essentially the same mechanism that accounts for the force in Fig. 2, except that in Fig. 2; many multipoles contribute as the spheres are much larger there. It can be seen from Fig. 5(b) that the single-sphere $l=1$ mode is split into a pair of attractive bonding mode and repulsive antibonding mode (note that the bonding mode at $3.50 \mathrm{eV}$ is difficult to see), respectively, at a frequency lower and higher than the single-sphere resonance.

For separation small compare to the particle size shown in Fig. 5(c) $\left[D /\left(2 r_{s}\right)=1.05\right]$, the modes are strongly interacting. ${ }^{23}$ One can observe in Fig. 5(c) a series of attractive and repulsive resonances. Unlike the previous cases where only the $l=1$ mode is excited, the higher order modes are also excited. The higher order antibonding modes can be excited because the quasieigenmodes of the bisphere are now hybridized modes that consist of individual nanospheres' modes of all $l$, which include a finite amount of the $l=1$ mode, and therefore can be excited by an incident plane wave. For the attractive bonding modes, they are excited owing to the retardation effect. Although the retardation effect is weak for such small and closely spaced particles, the force enhancement associated with bonding modes is strong owing to the steep slopes of the bonding mode bands, as shown in Fig. 1. As a result, the bonding mode induced attractive force is noticeable. The QSA is applied to calculate the resonant frequencies of the bonding modes and antibonding modes with $l=1,2,3$, and they are marked in Fig. 5(c) by downward and upward arrows, respectively. For $5 \mathrm{~nm}$ radius nanospheres, good agreement on the peak positions between the QSA and electrodynamics calculation has been achieved. However, despite the reasonable accuracy in predicting the peak positions, QSA cannot be applied to directly calculate the optical force, even for particles that are as small as $5 \mathrm{~nm}$ in radius and especially when they are closely spaced. For example, as QSA does not include the retardation effect, QSA will fail to predict all the attractive peaks in Fig. 5(c).

The total optical force $\vec{F}_{T}=\vec{F}_{2}+\vec{F}_{1}$ for the configuration of Fig. 5(c) is plotted in Fig. 5(d). There is a one to one correspondence between the optical binding peaks in Fig. 5(c) and the total optical force peaks in Fig. 5(d). The situation for other separation is similar. We note that the peak value of the total optical force is less than $1 \mathrm{pN}$. The total optical force is comparable with the resonant optical binding force only when the nanospheres are not in vicinity of each other.

In Fig. 6, the optical binding force for a pair of $30 \mathrm{~nm}$ radius nanospheres is plotted against the frequency. The po-
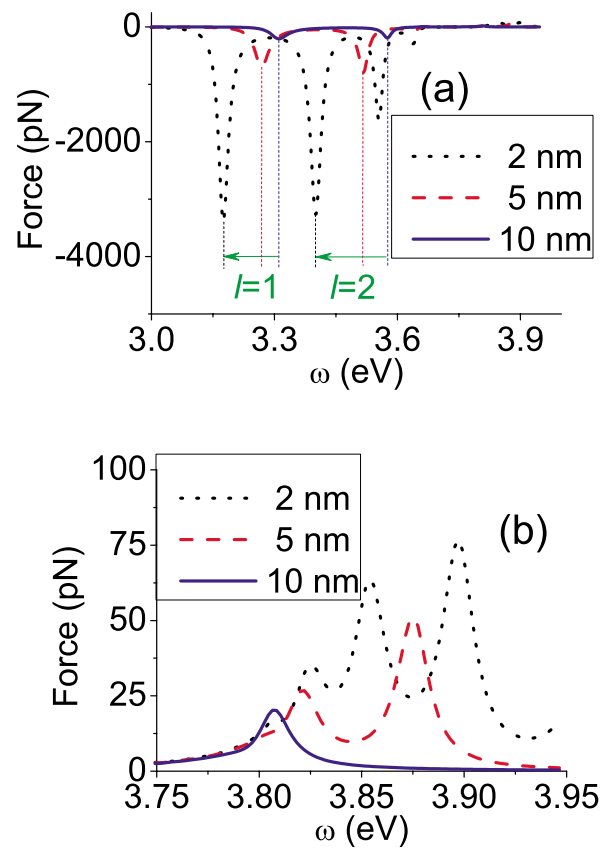

FIG. 6. (Color online) Optical binding force versus laser frequency for three separations. The polarization of the incident wave is perpendicular to the bisphere axis (i.e., an $x$-polarized and $z$-propagating plane wave). The surface-to-surface separation is 2 (black dotted line), 5 (red dashed line), and $10 \mathrm{~nm}$ (blue solid line). (a) The attractive bonding modes and (b) the repulsive antibonding modes.

larization is perpendicular to the bisphere axis. In Fig. 6(a), we clearly observe the attractive bonding modes to emerge at the lower frequency side of the spectrum, as predicted by Fig. 1(a). In Fig. 6(b), which is an enlarged session of the high frequency side of Fig. 6(a), we show that the repulsive antibonding modes emerge from the higher frequency side. We also note that the repulsive forces are much weaker than the attractive forces. The influence of the interparticle separation on the resonant force is depicted in Fig. 6(a) by considering different separations. As the separation decreases, the strength of the force increases and the resonance frequencies of the attractive (repulsive) modes shift to the red (blue). The redshifting of the bonding modes associated with $l=1$ and $l=2$ branches is illustrated by the arrows in Fig. 6(a). The optical forces for these two branches have increased by $\sim 17$ times as the surface-to-surface separation decreases from 10 to $2 \mathrm{~nm}$. In fact, within classical electrodynamics, upon further decrease in separation, the force will continue to rise and the frequency will continue to shift. In Fig. 7, wherein the polarization is parallel to the bisphere axis, we observe only the bonding modes at the lower frequency side; the higher frequency region that antibonding modes are supposed to emerge is featureless. The reason is that in the geometry we consider, the antibonding modes are orthogonal to the incident wave owing to symmetry, and as a result, the antibonding modes cannot be excited. Compared the two polarizations, the linewidth of the parallel polarization is wider than that of the perpendicular polarization, but the optical force associated with the perpendicular polarization is stronger. 


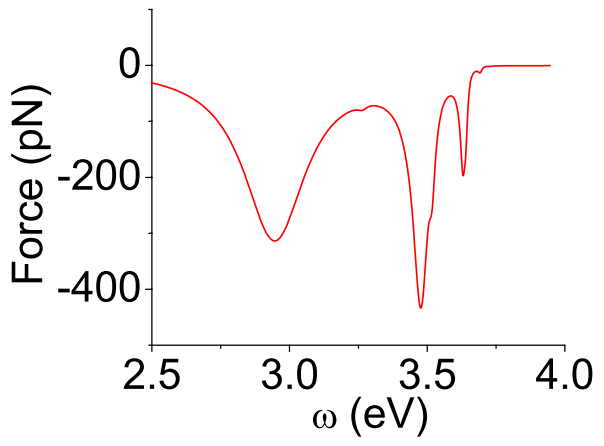

FIG. 7. (Color online) Optical binding force versus laser frequency. The polarization of the incident wave is parallel to the bisphere axis (i.e., a $z$-polarized and $x$-propagating plane wave). The surface-to-surface separation is $5 \mathrm{~nm}$.

\section{CONCLUSION AND DISCUSSION}

We found that for small particles, QSA is reasonably accurate when applied to calculate the resonance frequency, which, when combined with Eq. (2), can provide a qualitative understanding of the force between plasmonic particles. By using electrodynamics, it is shown that the bonding mode induced attraction can reach several nano-Newtons at the assumed intensity. This force can dominate over the competing van der Waals attraction when the particle separation is small, and it is also significantly stronger than the weight and thermal fluctuations. The antibonding mode induced repulsion is 2 orders of magnitude smaller than the bonding mode induced attraction; thus, it could play a role only at very high intensity.

In this paper, for illustrative purpose, we only consider using plane waves to excite the plasmonic resonances. Although we have not explicitly considered other type of incident waves, the profile of the incident wave should not be the key issue in the excitation of the plasmonic resonances. The most important issue is the incident frequency. If the incident frequency matches the resonance frequency of a mode, the mode can be excited as long as the symmetry of the incident wave is not orthogonal to that of the resonating mode.

By illuminating a pair of closely spaced metallic nanospheres with an incident light polarized along the bisphere axis, one would be able to create a hot spot in the small gap between the particles with enormous field intensity. Such hot spot could enormously enhance the signal of surface enhanced Raman spectroscopy, presumably to single molecule sensitivity. The separation between the particles is critical to the signal enhancement as a closer separation gives a much stronger signal. Nevertheless, it is difficult to control the separation to nanometric scale. Here, the SP induced optical force studied may provide a way to affect the separation and hence to control the field enhancement at the gap.

The bonding and antibonding optical forces are not unique to plasmonic structures. They were also previously reported in other systems. ${ }^{25,30-32}$ In the previous works, the resonant forces come from geometrical resonances of dielectric particles such as the Mie resonances and the whispering gallery modes. However, in this paper, what we consider is the SP induced optical force, which originates from the collective electronic excitation that is coupled to light. In fact, the plasmonic system is more strongly interacting compare to the dielectric systems. As a result, instead of simple splitting of modes as observed in dielectric system, the interaction for plasmonic system can create an attractive band at low frequency and a repulsive band at high frequency.

Finally, we stress that the physics of the resonant forces discussed here is rather general. Many of the observed features, including the formation of the attractive band and repulsive band, are based on a prediction of Eq. (2) that all attractive (repulsive) modes will bend downward (upward) as $D$ decreases. Since Eq. (2) is valid for any azimuthal number $m$, we shall expect similar physics for modes with any $m$. Furthermore, Eq. (2) can be generalized to handle a cluster of more than two dissimilar, arbitrarily shaped nanoparticles; consequently, we shall expect similar physics for other strongly interacting plasmonic systems as well.

\section{ACKNOWLEDGMENTS}

We thank Z. F. Lin for many helpful discussions. This work was supported by Hong Kong Central Allocation under Grant No. HKUST3/06C. Computation resources was supported by Shun Hing Education and Charity Fund. J. Ng was also supported by NSFC under Contract No. 10774028.

\footnotetext{
*Author to whom correspondence should be addressed; phchan@ust.hk

${ }^{1}$ K. Svoboda and S. M. Block, Opt. Lett. 19, 930 (1994).

${ }^{2}$ S. Sato, Y. Harada, and Y. Waseda, Opt. Lett. 19, 1807 (1994).

${ }^{3}$ P. M. Hansen, V. K. Bhatia, N. Harrit, and L. Oddershede, Nano Lett. 5, 1937 (2005).

${ }^{4}$ Y. Seol, A. E. Carpenter, and T. T. Perkins, Opt. Lett. 31, 2429 (2006).

${ }^{5}$ A. S. Zelenina, R. Quidant, and M. Nieto-Vesperinas, Opt. Lett. 32, 1156 (2007).

${ }^{6}$ J. R. Arias-Gonzalez and M. Nieto-Vesperinas, J. Opt. Soc. Am. A 20, 1201 (2003).
}

${ }^{7}$ R. Fuchs and F. Claro, Appl. Phys. Lett. 85, 3280 (2004).

${ }^{8}$ A. S. Zelenina, R. Quidant, G. Badenes, and M. NietoVesperinas, Opt. Lett. 31, 2054 (2006).

${ }^{9}$ B. A. Kemp, T. M. Grzegorczyk, and J. A. Kong, Phys. Rev. Lett. 97, 133902 (2006).

${ }^{10}$ H. Xu and M. Kall, Phys. Rev. Lett. 89, 246802 (2002).

${ }^{11}$ F. Svedberg, Z. Li, H. Xu, and M. Kall, Nano Lett. 6, 2639 (2006).

${ }^{12}$ A. J. Hallock, P. L. Redmond, and L. E. Brus, Proc. Natl. Acad. Sci. U.S.A. 102, 1280 (2005).

${ }^{13}$ K. Murakoshi and Y. Nakato, Adv. Mater. (Weinheim, Ger.) 12, 791 (2000). 
${ }^{14}$ P. Chu and D. L. Mills, Phys. Rev. Lett. 99, 127401 (2007); P. Chu and D. L. Mills, ibid. 100, 059901 (2008); P. Chu and D. L. Mills, Phys. Rev. B 77, 045416 (2008).

${ }^{15}$ Ref. 10 also studied the plasmonic attractive bonding forces by using electrodynamics. Our study differs from Ref. 10 in that Ref. 10 focuses on how the optical force affects the surface enhance Raman spectroscopy, whereas we focus on the properties of the optical force itself.

${ }^{16}$ J. Ng, Z. F. Lin, C. T. Chan, and Ping Sheng, Phys. Rev. B 72, 085130 (2005).

${ }^{17}$ Throughout this paper, the angular momentum truncation orders for the MS-MST calculations are taken as 43, 75, 76, 93, 94, and 96 for nanospheres of radii 5, 30, 50, 100, 150, and $200 \mathrm{~nm}$, respectively. We remark that since electrodynamics is not valid for near touching spheres, our result with smaller separation [e.g., $\left(D-2 r_{s}\right)<1 \mathrm{~nm}$ ] is less accurate.

${ }^{18}$ However, for extremely closely spaced particles, say when the gap between the particles is less than $1 \mathrm{~nm}$, in this case, the electrons on one of the sphere can actually leak to the other sphere. In other words, classical electrodynamics, and hence the MS-MST formalism, is not accurate.

${ }^{19}$ P. G. Kik, S. A. Maier, and H. A. Atwater, Phys. Rev. B 69, 045418 (2004).

${ }^{20}$ There is also a total optical force $\left(\vec{F}_{T}=\vec{F}_{2}+\vec{F}_{1}\right)$ that pushes the particle cluster as a whole; however, this total optical force is orders of magnitude smaller than the optical binding force and it is not our focus.

${ }^{21}$ M. M. Burns, J.-M. Fournier, and J. A. Golovchenko, Phys. Rev.
Lett. 63, 1233 (1989).

${ }^{22}$ E. Prodan, C. Radloff, N. J. Halas, and P. Nordlander, Science 302, 419 (2003).

${ }^{23}$ P. Nordlander, C. Oubre, E. Prodan, K. Li, and M. I. Stockman, Nano Lett. 4, 899 (2004).

${ }^{24}$ R. Ruppin, Phys. Rev. B 26, 3440 (1982).

${ }^{25}$ M. L. Povinelli, S. G. Johnson, M. Loncar, M. Ibanescu, E. J. Smythe, F. Capasso, and J. D. Joannopoulos, Opt. Express 13, 8286 (2005)

${ }^{26}$ J. N. Israelachvili, Intermolecular and Surface Forces, 2nd ed. (Academic, London, 1991).

${ }^{27}$ P. B. Johnson and R. W. Christy, Phys. Rev. B 6, 4370 (1972).

${ }^{28}$ This is because the terms that are quadratic in incident field equally contribute to both spheres and therefore do not contribute to the binding force, and the terms that are quadratic in the resonant scattered field are too small owing to the weak coupling between the particles.

${ }^{29}$ Owing to the azimuthal symmetry, modes with different $m$ are not mixed, but strictly speaking, modes with different $l$ are mixed. Nevertheless, at the present separation, the coupling is dominated by the modes with the same $l$.

${ }^{30}$ M. I. Antonoyiannakis and J. B. Pendry, Phys. Rev. B 60, 2363 (1999).

${ }^{31}$ M. L. Povinelli, M. Loncar, M. Ibanescu, E. J. Smythe, S. G. Johnson, F. Capasso, and J. D. Joannopoulos, Opt. Lett. 30, 3042 (2005).

${ }^{32}$ J. Ng, C. T. Chan, P. Sheng, and Z. F. Lin, Opt. Lett. 30, 1956 (2005). 